\title{
El lugar del conocimiento en la sociedad de la información La influencia de la tecnología en los procesos cognitivos
}

Uriel Rubén Cukierman`

\section{Resumen}

La tecnología se encuentra directamente vinculada al hombre y a su capacidad de adaptación al medio. Actualmente los nuevos desarrollos tecnológicos no siempre cumplen esta característica ya que, en muchos casos, son el resultado de acciones de marketing que imponen "supuestas" necesidades en la población. Muchos autores aseveran que la tecnología está modificando no sólo nuestros hábitos y costumbres más elementales, sino también nuestra manera de pensar e, inclusive, la estructura de nuestro cerebro. Existen pocos estudios aún que analicen cómo la tecnología influye o afecta a los procesos cognitivos asociados y derivados de su uso intensivo en la denominada Sociedad de la Información. A lo largo de este trabajo, se intentará abordar esta idea y llegar a algunas primeras conclusiones.

Palabras claves: Procesos cognitivos; Tecnología; Sociedad de la Información

\section{Abstract}

Technology is directly related to man and his ability to adapt to the environment. Currently the new technological developments do not always meet this characteristic because, in many cases, the result of marketing actions is what impose "alleged" needs to the people. Many authors assert that technology is changing, not only our most basic habits and customs, but also our thinking, and even the structure of our brain. There are still few studies that analyze how technology influences or affects cognitive processes associated to its intensive use in the so-called Information Society. Throughout this paper we attempt to address this idea and draw some initial conclusions.

Keywords: Cognitive processes; Technology; Information Society

Fecha de Recepción: junio 2012 | Fecha de aceptación: octubre 2012

- Universidad Tecnológica Nacional 


\section{Introducción}

La tecnología se encuentra, desde los orígenes de la humanidad, directamente vinculada al hombre y a su capacidad de adaptación al medio. Las primeras herramientas tecnológicas fueron desarrolladas por el hombre primitivo para proveerse de alimentos de manera más eficaz, protegerse del medio ambiente, enfrentar a los enemigos, etc. Este tipo de utilización de la tecnología es claramente instrumental, y tiene como objetivo optimizar los resultados de las acciones para las cuales se la desarrolla y/o utiliza. Esta aseveración es probablemente válida para casi toda la historia de la humanidad; aún cuando los recursos tecnológicos se iban complejizando y sofisticando, siempre tenían como objetivo mejorar las condiciones de vida del ser humano.

Sin embargo, en la actualidad los nuevos desarrollos tecnológicos no siempre cumplen con las características antes mencionadas ya que, en muchos casos, son el resultado de acciones de marketing que, de una $u$ otra manera, imponen "supuestas" necesidades en la población. Es muy significativa en tal sentido la frase del recientemente desaparecido Steve Jobs asegurando que "muchas veces, la gente no sabe lo que quiere hasta que se lo muestras" (Business Week, 1998). Más aún, existen muchos autores que aseguran que la tecnología está modificando no sólo nuestros hábitos y costumbres más elementales, sino también nuestra manera de pensar e, inclusive, la estructura de nuestro cerebro, como lo afirma Nicholas Carr en un libro de reciente publicación (Carr, 2010).

La utilización de la tecnología como apoyo al proceso de enseñar y aprender es un campo que, en la actualidad, se encuentra sumamente investigado y desarrollado; pero existen pocos estudios aún que analicen cómo la tecnología influye o afecta a los procesos cognitivos asociados y derivados de su uso intensivo en la denominada Sociedad de la Información (SI). A lo largo de este trabajo, se intentará abordar esta idea y llegar a algunas primeras conclusiones que, necesariamente, deberán seguir siendo estudiadas y analizadas en profundidad y a posteriori.

\section{Conocimiento, memoria y educación}

La idea de almacenar todo el conocimiento humano sobre un soporte tecnológico tiene un antecedente significativo en el Siglo XVIII. Dicha idea, que surgió bajo la influencia de la Ilustración, es conocida como Enciclopedismo y se trataba de un movimiento filosófico y pedagógico que promovió la creación de una obra que contuviera todo el conocimiento, la que dio en llamarse "Enciclopedia", y fue publicada en Francia por Diderot y D'Alembert entre los años 1751 y 1772 (Figura 1) (Bibliothèque nationale de France). 
La educación enciclopedista, basada en los contenidos y en su transmisión desde un maestro erudito a un alumno ignorante, surgió entonces como una consecuencia casi natural de dicho movimiento. La buena memoria resultaba, en ese escenario, un elemento virtuoso debido a que representaba la internalización de un universo de conocimiento externo. "Los pueblos antiguos y medievales le brindaron admiración a la memoria. Sus más grandes genios fueron descriptos como la gente de recuerdos superiores," escribe Mary Carruthers, la autora de dos libros sobre la historia de las técnicas memorísticas. (Foer, 2011) Aún hoy en día buena parte del sistema educativo está basado, lamentablemente, en dicha escuela. La concepción constructivista de la educación sostiene, en cambio, que el aprendizaje escolar es el resultado de un complejo proceso de intercambios funcionales que se establecen entre tres elementos: el alumno que aprende, el contenido que es objeto de aprendizaje y el profesor que ayuda al alumno a construir significados y a atribuir sentido a lo que aprende (Coll, 1996).

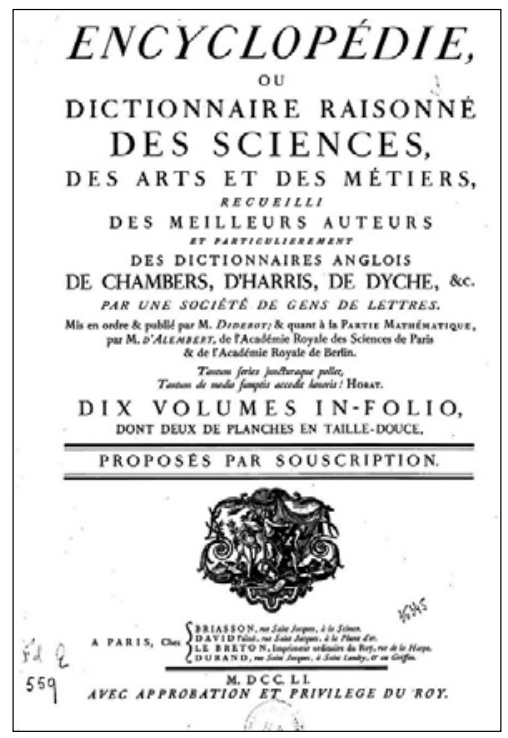

Figura 1

Pero en cualquier teoría de la educación que se utilice, la relación entre conocimiento, memoria y educación es un tema central y pertinente; se puede decir que adquirir conocimiento es educarse y que el conocimiento se obtiene, básicamente, por la acción de la percepción y la memoria (Guerra González, 2005). Ahora bien, la pregunta es entonces si la utilización de soportes tecnológicos externos para el conocimiento libera al individuo de la necesidad de incorporarlos en su propia memoria personal. Una respuesta 
positiva a esta pregunta sólo suena posible en un relato de ciencia ficción. Sin embargo, es bien conocida la frase atribuida a Albert Einstein con respecto a que no es necesario memorizar lo que se puede encontrar en un libro. Esta frase no debe ser interpretada literalmente, sino en función de un uso efectivo de la memoria. En efecto, la falta total de memoria, sólo verificada en casos patológicos, no es el ideal de ninguna persona y más bien puede asegurarse que la memoria, en cuanto almacenamiento de datos, es una habilidad que puede ser ejercitada y desarrollada hasta niveles superlativos (Foer, 2011), pero que por sí misma no se traduce en conocimiento y, mucho menos, en educación. Como se dijo anteriormente, aprender algo consiste en construir significados y atribuir sentido a partir de un conocimiento o contenido.

La historia del almacenamiento del conocimiento en medios físicos externos - tecnología - se inicia ya con los más antiguos ancestros humanos y sus pinturas rupestres. Se desarrolla de manera muy significativa con el libro, muy especialmente con la invención de la imprenta, y alcanza un grado supremo en la actualidad con las Tecnologías de la Información y la Comunicación (TICs). En efecto, los dispositivos electrónicos actuales conectados a Internet brindan acceso cuasi inmediato a información y datos prácticamente ilimitados. Wikipedia y Google representan hoy en día lo que la "Enciclopedia" de Diderot y D'Alembert significó para los hombres del Siglo XVIII. "Había una vez en que la memoria estaba en la raíz de toda la cultura, pero en los últimos treinta milenios desde que los humanos comenzaron a pintar sus memorias en las paredes de las cavernas, hemos suplantado gradualmente nuestra propia memoria natural con una vasta estructura de ayudas externas a la memoria - un proceso que se ha acelerado exponencialmente en años recientes. Imagine despertándose mañana y descubriendo que toda la tinta del mundo se ha vuelto invisible y que todos nuestros bytes han desaparecido. Nuestro mundo se desmoronaría inmediatamente. Literatura, música, leyes, política, ciencia, matemática: Nuestra cultura es un edificio construido en base a recuerdos externalizados" (Foer, 2011).

Por otro lado, y en contraposición, la imposibilidad de olvidar también constituye un signo de la modernidad tecnológica. De la misma manera que "Funes el memorioso" (Borges, 1978), aquel recordado personaje de Borges que, como resultado de un accidente, había perdido su habilidad de olvido, hoy en día todo lo que se hace "on line" queda, de una u otra manera, registrado en el ciberespacio. Más aún para aquellos que utilizan las redes sociales asiduamente. Este tema podría ser motivo de más de un estudio psico-sociológico que excede los alcances de este trabajo. 


\section{Los procesos cognitivos y la tecnología}

Cognición deriva de la palabra griega gnome $(\gamma v \omega ́ \mu \eta)$ que significa facultad de conocer, entendimiento, razón, juicio. La misma palabra se utiliza para aquella famosa y célebre sentencia que sintetiza el espíritu griego, la muy conocida

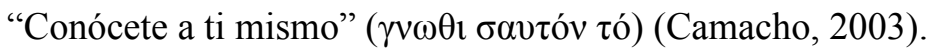

Desde un punto de vista científico la cognición puede ser definida como el acto de conocer, y la psicología cognitiva es el estudio de todas las actividades humanas relacionadas con el conocimiento. Estas actividades incluyen la atención, la creatividad, la memoria, la percepción, la resolución de problemas, el pensamiento y el uso del lenguaje (Neisser).

La relación existente entre la tecnología y los procesos cognitivos surge entonces en forma directa, especialmente en la actualidad. Hoy en día varias de las actividades listadas en el párrafo anterior se realizan a través de medios electrónicos y, a su vez, dichos medios condicionan y modelan la forma en que se accede al conocimiento. Por ejemplo, la atención: La capacidad de sostener la atención a una determinada actividad durante un cierto tiempo está en la actualidad minada por la gran cantidad y variedad de estímulos externos que se presentan en cualquier momento y lugar. Hace no mucho tiempo, leer o estudiar eran tareas que se solían realizar en bibliotecas o ambientes relativamente asépticos en lo que se refiere a interferencias sonoras y/o auditivas. Sin entrar a analizar la eficacia relativa de las modernas costumbres respecto de las anteriores, es muy frecuente encontrar a los jóvenes, y otros no tanto, leyendo o estudiando sometidos voluntariamente a una gran diversidad de estímulos. Es bien conocido el término inglés multitasking o multitarea con que se caracteriza a los jóvenes actuales que son capaces, de una manera sorprendentemente eficaz, de ver la televisión, escuchar música, "chatear" con sus amigos y realizar una tarea escolar, todo en forma simultánea. Las dudas acerca de si estas conductas son beneficiosas o perjudiciales son motivo de muchos estudios. De lo que no cabe duda es de que modifican la atención y el acceso al conocimiento.

Sobre la memoria y cómo ésta se ve afectada por la tecnología, ya se ha descrito suficientemente en la sección anterior. La percepción del mundo circundante está, cada vez más, afectada por la tecnología. Si bien los medios audiovisuales existen desde hace décadas, sólo en la actualidad su ubicuidad, universalidad y variedad representan una alternativa plausible a la percepción directa de la realidad. Se puede afirmar que los medios masivos han dejado de ser externos a los jóvenes y se han incorporado a las experiencias personales, familiares y escolares, se han hecho parte de su sociabilidad y han transformado, además, los modos de percepción (Castillo Barragán, 2006).

A algunas personas podría parecerles exagerado suponer que el uso de la tecnología afecta al pensamiento, pero existen numerosas evidencias en tal sentido; 
más aún, se han realizado estudios que demuestran que la mente se modifica al ser expuesta de manera frecuente a los estímulos digitales. "La actual explosión de la tecnología digital no sólo está cambiando la forma en que vivimos y nos comunicamos, sino que también está alterando rápida y profundamente nuestros cerebros. La exposición diaria a la tecnología avanzada - computadoras, teléfonos inteligentes, videojuegos, motores de búsqueda como Google y Yahoo - estimula la alteración de las células cerebrales y la liberación de neurotransmisores, fortaleciendo gradualmente nuevas conexiones neuronales en nuestro cerebro al tiempo que debilitando las antiguas. Debido a la actual revolución tecnológica, nuestros cerebros se están desarrollando en este momento a una velocidad como nunca antes" (Small \& Vorgan, 2008). Por su parte, y en el mismo sentido, Carr plantea que el pensamiento humano ha sido moldeado a través de los siglos por las llamadas "herramientas de la mente", desde el alfabeto, pasando por los mapas, los textos impresos, el reloj y hasta la computadora. Luego avanza en su planteo, basado en recientes descubrimientos en el campo de la neurociencia, aseverando que las evidencias históricas y científicas revelan que nuestros cerebros se modifican como consecuencia de nuestras experiencias y que las tecnologías que utilizamos para encontrar, almacenar y compartir información pueden "reencaminar" nuestras redes neuronales. Sostiene también que los escasos instantes de atención que demanda la toma de decisiones al seleccionar hipervínculos en una página Web, van en contra del poder cognitivo necesario para la lectura y que los estímulos simultáneos que provoca Internet en nuestros sentidos, reducen la capacidad de retención en la memoria, afectando finalmente la habilidad y la paciencia para manejar las relaciones humanas en toda su complejidad (Cukierman, 2011)

Por último, y con referencia al uso del lenguaje, las evidencias acerca de cómo éste se ve afectado por la tecnología pueden verse desde el mero envío de un mensaje de texto (SMS) entre celulares o en la limitación respecto de la cantidad de caracteres habilitados para la publicación de un comentario vía Twitter. Para mucha gente, la siguiente secuencia de caracteres pso tngo mvid fmily sbdo bss wpos kuntm $\boldsymbol{n}$ chat podría significar algo absolutamente ilegible, pero alguien acostumbrado a las abreviaciones utilizadas en los SMS lo leería como paso, tengo movida familiar el sábado. Besos guapos. Cuéntame en el chat. "La forma en que las alteraciones en las lenguas se expanden se ven favorecidas por los propios medios en los cuales surgen y se hacen de uso cotidiano. [...] Una cuestión es segura. El lenguaje, tal como lo conocemos, y las lenguas, tal como sobreviven al día de la fecha, no están muriendo. Al contrario, se están nutriendo, están creciendo, evolucionando. Impregnadas en el proceso de creatividad, ingenio, pereza por qué no, e incluso economía. [...] Esta realidad no es definitiva, porque al mismo tiempo que las tecnologías avancen, lo hará el lenguaje dentro de los entornos virtuales que perduren, evolucionen o se inventen." (Parrilla, 2008) 


\section{La Sociedad de la Información y el Conocimiento}

El concepto de SI ha sido tratado por diversos autores, en diferentes momentos y con variadas perspectivas, aunque suele reconocerse a Daniel Bell como el primero en hacerlo de una manera formal y justificada. En su planteo asegura que la sociedad post-industrial es una sociedad del conocimiento en un doble sentido: en primer lugar porque las fuentes de la innovación se derivan de la investigación y el desarrollo y, en segundo lugar, el peso de la sociedad - medido en términos de una mayor proporción del Producto Bruto Interno (PBI) y una mayor parte del empleo - se encuentra crecientemente en el campo del conocimiento (Bell, 1973).

Posteriormente, a mediados de los noventa, la expresión SI vuelve al centro de la escena a partir de su inclusión en la agenda del G7 $7^{1}$ y, más específicamente, a través de la organización del Congreso de la Sociedad de la Información en Febrero de 1995 en Bruselas. Unos años más tarde, la Unión Internacional de Telecomunicaciones (UIT) y la Organización de la Naciones Unidas (ONU), eligen esta expresión como identificador de las cumbres mundiales a realizarse en 2003 y 2005 en Génova y Túnez respectivamente².

Más allá del origen de la expresión, parte del desafío, que al mismo tiempo se presentaba como una oportunidad consistía, según la visión imperante en ese momento, en que las grandes economías estaban pasando de un modelo de producción industrial a otro más centrado en el conocimiento. Se planteaba entonces que la mejora de la eficiencia en los procesos productivos llevaría a una disminución de los puestos de trabajo, la que sería compensada con la aparición de nuevas actividades y más oportunidades (Funk \& Esteban, 1996).

En la actualidad, y en particular ésta es la visión de quien escribe este documento, se tiende a hablar de la Sociedad de la Información y el Conocimiento (SIC) o, más simplificadamente, Sociedad del Conocimiento (SC). En realidad, el concepto de información sólo refiere a datos que hayan sido ordenados por categorías y planes de clasificación u otras pautas; en cambio conocimiento significa información que ha sido depurada dándole forma de afirmaciones más generales (Toffler, 1990). Como se mencionaba previamente en este mismo texto, hoy existe una sobreabundancia de información disponible, especialmente a través de Internet, pero es sólo eso, información. Para que se transforme en conocimiento deberá ser primero ubicada, seleccionada, clasificada e incorporada en el marco de un significado que le dé sentido (Cukierman, Rozenhauz, \& Santángelo, 2009). En realidad, los cambios

1. Grupo de los países más industrializados, actualmente G8 (Alemania, Canadá, Estados Unidos, Francia, Italia, Japón, Reino Unido y Rusia)

2. http://www.itu.int/wsis/index.html 
han sido tantos y tan significativos en las últimas dos décadas que es posible postular que ya vivimos en una nueva Era. "De hecho hubo quienes plantearon que esta nueva Era comenzó con hitos como el fin de la Segunda Guerra Mundial (Era Atómica), la llegada del hombre a la Luna (Era Espacial), o la caída del Muro de Berlín (Era Global), entre otros hechos significativos del Siglo XX. [...] Seguramente podremos encontrar muchas otras más denominaciones para la Era en la que estamos viviendo pero, cada vez tiende más a generalizarse la visión de que son las nuevas tecnologías digitales, las que significan un punto de inflexión en los usos y costumbres de la mayor parte de la humanidad." (Cukierman \& Virgili, La educación en el siglo XXI: Los desafíos de la Era Digital, 2010). Esta afirmación conduce entonces, nuevamente, al eje de este trabajo y se puede reafirmar en consecuencia que los procesos cognitivos se ven, en la actualidad, fuertemente influenciados por la tecnología y que, a su vez, el nuevo conocimiento genera nuevas tecnologías, constituyendo así una especie de ciclo realimentado que se va acelerando e incrementando en magnitud de manera exponencial.

Resulta imperativo entonces, realizar estudios e investigaciones que permitan conocer a fondo las implicancias de dicho proceso y evitar que siga un curso que esté guiado por su propia inercia o, lo que podría ser peor aún, por las denominadas "fuerzas del mercado". Las universidades y centros de investigación de todo el mundo deberán poner todo el esfuerzo y dedicación en esta tarea que podrá dar a los gobernantes los conocimientos para actuar de manera sabia y equilibrada y hacer que los desafíos antes planteados se resuelvan de una manera beneficiosa para la humanidad.

\section{Bibliografía, referencias y notas}

Business Week. (25 de Mayo de 1998). Recuperado el 8 de Enero de 2012, de http:// www.businessweek.com/archives/1998/b3579156.arc.htm

Bell, D. (1973). The coming of post-industrial society: a venture in social forecasting. New York: Basic Books.

Bibliothèque nationale de France. (s.f.). Recuperado el 8 de Enero de 2012, de http://gallica.bnf.fr/ark:/12148/bpt6k5785794x

Borges, J. L. (1978). Funes el memorioso. En Ficciones (pág. 122). Madrid: Alianza. Camacho, J. M. (2003). Fundación Foro. Recuperado el 9 de Enero de 2012, de http://www.diproredinter.com.ar/articulos/pdf/acbcognitivo.pdf 
Carr, N. G. (2010). The Shallows: What the Internet Is Doing to Our Brains. W.W. Norton \& Co.

Castillo Barragán, C. (2006). Medios masivos de comunicación y su influencia en la educación. Odiseo, Año 3(6).

Coll, C. (1996). Constructivismo y educación escolar: ni hablamos siempre de lo mismo ni lo hacemos siempre de la misma perspectiva epistemológica. Anuario de Psicología - Universitat de Barcelona(69), pág. 175.

Cukierman, U. R. (2011). Comentario sobre el libro "Superficiales: ¿Qué está haciendo Internet con nuestras mentes?". RUEDA(8).

Cukierman, U. R., \& Virgili, J. M. (2010). La educación en el siglo XXI: Los desafíos de la Era Digital. En La tecnología educativa al servicio de la educación tecnológica: Experiencias e investigaciones en la UTN. Buenos Aires: edUTecNe.

Cukierman, U., Rozenhauz, J., \& Santángelo, H. (2009). Tecnología Educativa: Recursos, modelos y metodologías. Buenos Aires: Pearson.

Foer, J. (2011). Moonwalking with Einstein: the art and science of remembering everything. New York: The Penguin Press.

Funk, H., \& Esteban, J. A. (1996). Autopistas de la Información: El reto del Siglo XXI. Madrid: Complutense.

Guerra González, M. (Diciembre de 2005). Una reflexión filosófica sobre la relación entre conocimiento, educación y ética. La Revista Venezolana de Educación (Educere), 9(31), 579-588.

Neisser, U. (s.f.). Cognitive psychology. Recuperado el 9 de Enero de 2012, de Grolier Multimedia Encyclopedia: http://ww2.justanswer.de/uploads/ Kereya/2009-07-17_054616_Grolier_Online_Cognitive.pdf

Parrilla, E. A. (2008). Alteraciones del lenguaje en la era digital. Comunicar, págs. 131-136.

Small, G., \& Vorgan, G. (Octubre de 2008). Scientific American Mind. Recuperado el 5 de Abril de 2009, de http://www.sciam.com/article.cfm?id=your-ibrain Toffler, A. (1990). El cambio del poder. Barcelona: Plaza \& Janés. 
